\title{
Performance evaluation of decode and forward cooperative diversity systems over nakagami-m fading channels with non-identical interferers
}

\author{
Mamoun F. Al-Mistarihi ${ }^{1}$, Rami Mohaisen ${ }^{2}$, Khalid A. Darabkh ${ }^{3}$ \\ ${ }^{1,2}$ Electrical Engineering Department, Faculty of Engineering, Jordan University of Science and Technology, Jordan \\ ${ }^{3}$ Computer Engineering Department, Faculty of Engineering and Technology, The University of Jordan, Jordan
}

\begin{tabular}{l} 
Article Info \\
\hline Article history: \\
Received Oct 2, 2019 \\
Revised Apr 4, 2020 \\
Accepted Apr 14, 2020 \\
\hline Keywords: \\
Cooperative wireless networks \\
Decode-and-forward \\
Incremental relaying \\
Maximal ratio combiner \\
Nakagami-m fading channel \\
Non-identical interferers \\
\hline
\end{tabular}

\begin{abstract}
Incremental relaying (IR) was developed to overcome the problems facing regular cooperative relaying methods. Out of the regular methods there is fixed relaying, in which, the relay transmits the source's signal to the destination without considering the state of the channel. On the contrary, adaptive relaying techniques, including IR, are becoming popular among researchers nowadays; since they efficiently utilize the channel. In this paper, we studied the performance of a two-hop IR system that has decode and forward (DF) relays. Moreover, this system was analyzed over Nakagami-m fading channels, with the presence of various interferers positioned near the destination. As a result, the system suffered from co-channel interference. Remarkably, in this work, formulas were driven for the outage probability (OP) and the bit error rate (BER), and the assumptions were checked numerically.
\end{abstract}

Copyright $(9) 2020$ Institute of Advanced Engineering and Science. All rights reserved.

\section{Corresponding Author:}

Mamoun F. Al-Mistarihi,

Electrical Engineering Department, Faculty of Engineering,

Jordan University of Science and Technology,

P.O. Box 3030, Irbid 22110, Jordan.

Email: mistarihi@just.edu.jo

\section{INTRODUCTION}

High data rates are required to serve multimedia operations in modern wireless communication systems [1-3]. Interestingly, such systems need to be designed carefully, and studies are encouraged to plan the link budget [4-7]. Designing modern communication systems to provide wide coverage and high data rates cannot be done only with direct transmissions [8-11]. Therefore, studies considered multi-hop relaying in the designing of these systems to overcome the coverage problem [12]. Multi-hop relaying is considered because of the non-linear relation between the distance and the propagation loss, which reduces the end-to-end attenuation and relaxes the link budget [12]. Interestingly, wireless and mobile networks use traditional relaying in its place of microwave links and satellite relays $[1,12]$.

Interestingly, cooperative diversity considers using several relay nodes in transmitting the signal towards the destination, which guarantees achieving diversity gain. The concept of cooperative diversity has two features [1, 12]. First of all, transmitting to the intended node and to other neighboring nodes (i.e., broadcasting the signal). Second of all, in cooperative diversity we can consider the antennas of distributed nodes as a multiple-antenna system. Therefore, as a conclusion, cooperative diversity mixes conventional relaying with multiple-antenna systems.

The source's signal is delivered to the destination via intermediate nodes that implement cooperative diversity methods. Such methods support distributed spatial diversity, widen the coverage area, and lengthen the node's battery life [13]. When a relay is used, there will be two phases for the transmission. First, an information packet is sent by the source to the destination directly and to the relay (for non-direct 
transmission). Second, separate transmissions, of the information packet, will take place at the source and at the relay towards the destination with a prespecified multiple access technique.

Multipath fading can be prevented by cooperative diversity [14], in which, the antennas of the nodes act as a virtual antenna array. This is done in order to avoid having many antennas on each node, for limitations like size and complexity. Cooperative digital relaying protocols have the problem of error propagation in the relay destination link. To solve this problem, there are two scenarios. First, the source-relay's Channel State Information (CSI) would be known to the destination [14], or, the second, the relay would transmit the source's signal depending on its quality [15]. The second solution wastes the spectrum resource in half-duplex relaying.

Fixed relaying methods represent one form of cooperative diversity in which a pre-deterministic manner governs the relaying process of intermediate nodes [13]. These methods are easy to implement but they don't utilize the channel efficiently. Amplify-and-Forward (AF) and the DF relaying techniques were explained in [16-26]. On the other hand, in [27, 28], the authors derived the BER and the OP of Rayleigh channels with AF fixed relaying. Moreover, AF relaying performance in Rayleigh fading environment was studied in terms of OP and symbol error probability (SEP) in [29].

As aforementioned, the utilization of channel resources is low in fixed relaying, thus, Incremental Relaying (IR) is employed in modern wireless communication systems. IR systems use the relay only when needed, thus enhances the channel utilization. As an application, authors of [30, 31] have studied a DF-IR system with one relay through a Rayleigh fading channel. As for their contribution, they derived formulas for the BER and the OP. Authors of $[32,33]$ have derived the formulas for the BER and the OP of a two-hop DF-IR via Nakagami-m and mixed fading channels with existence of several $L$ equal interferers placed close to the destination.

In this paper, we propose a full examination of a DF-IR system that suffers from co-channel interference due to Nakagami-m fading along with some L unique interferers placed near the destination. A sum of distinguishable and independent Rayleigh random variables is used to represent the interferers in the system. In specific, we properly examine the relay-based DF cooperative diversity system's performance with the system consideration of Nakagami-m fading channels with distinguishable interferers near the destination. Preliminary results of this work have been presented in [34].

Besides, the following bullet points provide a summary of our contributions:

1. Coming into both the Cumulative Distributed Function (CDF) and the Probability Density Function (PDF) of the spontaneous Signal to Interference Ratio (SIR) at the combiner's output and consequently using them in formulating the BER and the OP.

2. Attaining tight formulas for the system's BER and OP with the existence of several L distinguishable interferers close to the destination. The destination-interferer fading channels are assumed to be the sum of unique and independent Rayleigh random variables.

This work is formed as the following; Section 2 displays our proposed system model. Section 3 has the analysis of the system's performance and the derivation of the BER and OP expressions. Section 4 shows the results with discussions. In the end, section 5 concludes this work.

\section{SYSTEM MODEL}

Figure 1 shows a single channel that has three independent Nakagami-m coefficients; the source-destination $h_{S, D}$, the relay-destination $h_{R, D}$ and the source-relay $h_{S, R}$. A feedback sent by the destination requesting the source's signal from the relay is called negative feedback. This feedback is triggered, towards the relay and the source, if the signal of the source wasn't received clearly in the first time slot. In the following time slot, the signals received from the relay and ones received from the source will be combined at the destination using maximal ratio combiner (MRC) to enhance the received signal. An additive white gaussian noise (AWGN), with a variance equal to 1, is assumed to exist at the destination. There exists an antenna per node along with several L interferers near the destination that introduce co-channel interference. Remarkably, the interferers links are assumed to be Rayleigh random variables (unique and independent). Moreover, the sum of the Rayleigh random variables signifies the channel holding the aforementioned connections. Time division multiple access is the multiple access technique adopted in this paper.

In the first time slot, the signal is broadcasted by the source. Consequently, in the second time slot, a decision is made by the destination to determine whether it needs a relay or not. If the relay is not needed, (or we have a successful Direct Transmission (DT)), a positive feedback would be transmitted throughout two paths, (the relay-destination and the source-destination), acquiring another signal from the source in the following time slot. 


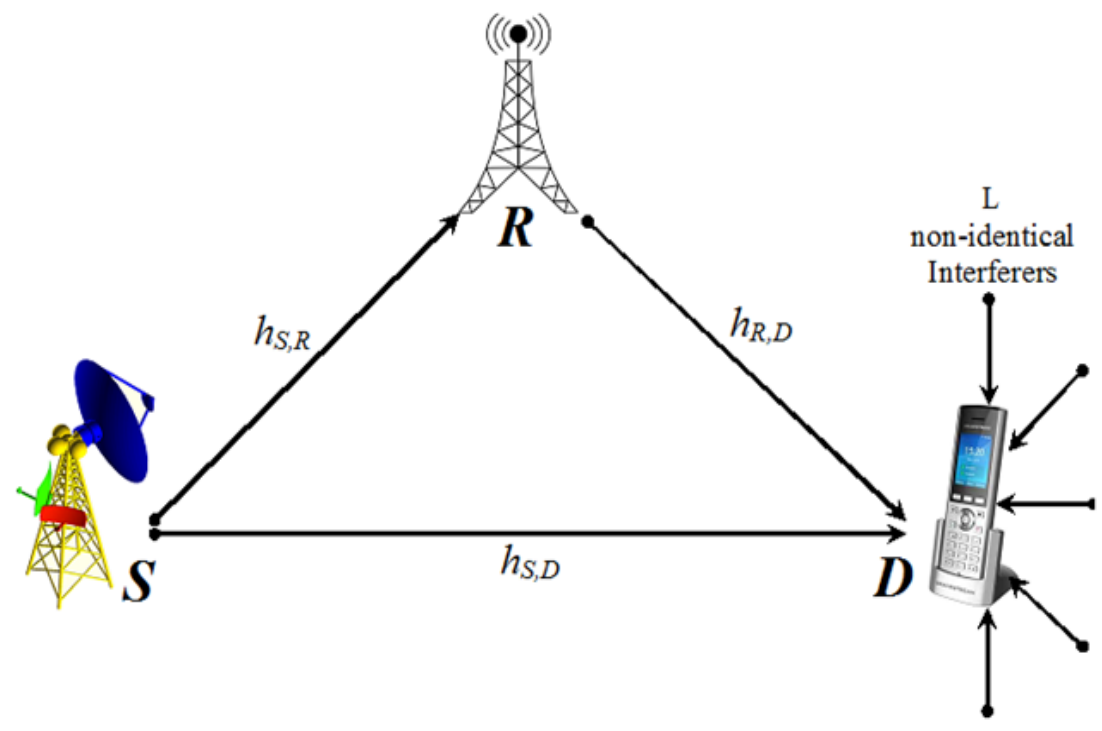

Figure 1. Cooperative diversity system with several L distinguishable Interferers placed close to the destination

Considering the events described above, the signals in the first time slot are going to be examined at the destination and at the relay as shown on (1) and (2):

$$
\begin{aligned}
& y_{S, D}\left(t_{1}\right)=h_{S, D} \sqrt{E_{S}} x\left(t_{1}\right)+n_{1}\left(t_{1}\right)+I\left(t_{1}\right) \\
& y_{S, R}\left(t_{1}\right)=h_{S, R} \sqrt{E_{S}} x\left(t_{1}\right)+n_{2}\left(t_{1}\right)
\end{aligned}
$$

where $y_{S, D}\left(t_{1}\right)$ and $y_{S, R}\left(t_{1}\right)$ declare the two signals received at the destination and the relay and $E_{S}$ is the energy of the source signal. $x\left(t_{1}\right)$ is the signal of the source in the first time slot. $n_{1}\left(t_{1}\right)$, and $n_{2}\left(t_{1}\right)$ are the AWGN symbols. $I\left(t_{1}\right)$ represents the interferers' effects in the first time slot.

If a DT was not accomplished, a negative feedback is sent by the destination towards the source and the relay, which represent a query for the relay's help. The relay will decode the source's signal, re-encode it, and finally transmit it to the destination in the second time slot. Telling the events described above in equation format can be done by expressing the destination's received signal in the second time slot in (3):

$$
y_{R, D}\left(t_{2}\right)=h_{R, D} \sqrt{E_{s}} x_{r}\left(t_{2}\right)+n_{3}\left(t_{2}\right)+I\left(t_{2}\right)
$$

where $x_{r}\left(t_{2}\right)$ is the relay's re-encoded signal, $I\left(t_{2}\right)$ is the interferers' effects within the second time slot. Interestingly, the source-destination path's outage depend on comparing the path's SNR versus $\gamma_{o}$, which signifies the minimum threshold that lets the destination correctly handle the source's signal from the DT.

\section{PERFORMANCE ANALYSIS}

We bypassed the effect of the noise at the destination in our analysis; because it is not comparable with the co-channel interference. In other words, we will use SIR instead of signal-to-interference-and-noiseratio (SINR). In the subsequent text, the derivation of the destination SIR's PDF and CDF from both the relay-destination and the source-destination paths is proposed.

\subsection{PDF of SIR's $\boldsymbol{\eta}_{S, D}$ and $\boldsymbol{\eta}_{R, D}$}

The SIR's $\left(\eta_{S, D}\right)$ PDF in the source-destination's link is calculated as the following:

$$
f_{\eta_{S, D}}\left(\eta_{S, D}\right)=\frac{(m) !}{\Gamma(m)} \sum_{k=1}^{L} \pi_{k}\left[\frac{\lambda_{S, D} \eta_{S, D}^{m-1}}{\left(\eta_{S, D}+\lambda_{S, D}\right)^{m+1}}\right]
$$


where $\lambda_{S, D}=\left(\frac{\overline{\frac{\gamma S, D}{D}}}{m \overline{\gamma_{k, 1}}}\right), 1$ is used to refer to the first time slot, $\pi_{k}=\prod_{\substack{i=1 \\ i \neq k}}^{L} \frac{\overline{\gamma_{k}}}{\overline{\gamma_{k}}-\overline{\gamma_{l}}}, \mathrm{~L}$ is the number of interferers.

The SIR's $\left(\eta_{R, D}\right)$ PDF in the relay-destination's link is calculated as the following:

$$
f_{\eta_{R, D}}\left(\eta_{R, D}\right)=\frac{(m) !}{\Gamma(m)} \sum_{k=1}^{L} \pi_{k}\left[\frac{\lambda_{R, D} \eta_{R, D}^{m-1}}{\left(\eta_{R, D}+\lambda_{R, D}\right)^{m+1}}\right]
$$

where $\quad \lambda_{R, D}=\left(\frac{\overline{\gamma_{R, D}}}{m \overline{\gamma_{k, 2}}}\right), 2$ is used to refer to the second time slot.

Proof:

The SNRs of the source-destination and the relay-destination paths, $\gamma_{S, D}$ and $\gamma_{R, D}$ respectively, are exponentially distributed. Moreover, the interferers fading channels' SNR $\left(\gamma_{b}=\sum_{k=1}^{L} \gamma_{k}\right)$ is assumed to be the sum of unique and independent exponential random variables. Thus, the SIR's $\left(\eta_{S, D}\right) \operatorname{PDF}$ is processed as [35]:

$$
f_{\eta_{S, D}}\left(\eta_{S, D}\right)=\int_{0}^{\infty} \gamma_{b} f_{\gamma_{S, D}}\left(\gamma_{b} \eta_{S, D}\right) f_{\gamma_{b}}\left(\gamma_{b}\right) d \gamma_{b}
$$

and the SIR's $\left(\eta_{R, D}\right)$ PDF is computed as:

$$
f_{\eta_{R, D}}\left(\eta_{R, D}\right)=\int_{0}^{\infty} \gamma_{b} f_{\gamma_{R, D}}\left(\gamma_{b} \eta_{R, D}\right) f_{\gamma_{b}}\left(\gamma_{b}\right) d \gamma_{b}
$$

The PDFs of $\gamma_{S, D}, \gamma_{R, D}$ and $\gamma_{b}$ are denoted as:

$$
\begin{aligned}
& f_{\gamma_{S, D}}\left(\gamma_{S, D}\right)=\frac{m^{m} \gamma_{S, D} m-1}{\overline{\gamma S, D}^{m} \Gamma(m)} \exp \left(-\frac{m \gamma_{S, D}}{\overline{\gamma_{S, D}}}\right), \gamma_{S, D}>0 \\
& f_{\gamma_{R, D}}\left(\gamma_{R, D}\right)=\frac{m^{m} \gamma_{R, D} m-1}{{\overline{\gamma_{R, D}}}^{m} \Gamma(m)} \exp \left(-\frac{m \gamma_{R, D}}{\overline{\gamma_{R, D}}}\right), \gamma_{R, D}>0 \\
& f_{\gamma_{b}}\left(\gamma_{b}\right)=\sum_{k=1}^{L} \frac{\pi_{k}}{\overline{\gamma_{k}}} e^{\left(-\frac{\gamma_{b}}{\gamma_{k}}\right)}, \gamma_{b}>0
\end{aligned}
$$

while the average SNR of each destination-interferer's channel is represented by $\overline{\gamma_{k}}$.

Integral (6) can be solved by using $[36,(3.351 .3)]$ to get the PDF of the SIR for the source-destination's link in (4). Similarly, integral (7) is solved using the same equation to obtain the SIR's PDF of the relay path in (5).

\subsection{CDF of SIR's $\eta_{S, D}$ and $\eta_{R, D}$}

The SIR's $\left(\eta_{S, D}\right)$ CDF in the source-destination's link is calculated as the following:

$$
F_{\eta_{S, D}}\left(\eta_{S, D}\right)=\frac{(m-1) !}{\Gamma(m)} \sum_{k=1}^{L} \pi_{k}\left(\frac{\eta_{S, D}}{\lambda_{S, D}}\right)^{m}{ }_{2} 1\left(m+1, m ; m+1 ;-\frac{\eta_{S, D}}{\lambda_{S, D}}\right)
$$

The SIR's $\left(\eta_{R, D}\right)$ CDF in the relay-destination's link is provided as the following:

$$
F_{\eta_{R, D}}\left(\eta_{R, D}\right)=\frac{(m-1) !}{\Gamma(m)} \sum_{k=1}^{L} \pi_{k}\left(\frac{\eta_{R, D}}{\lambda_{R, D}}\right)^{m}{ }_{2} 1\left(m+1, m ; m+1 ;-\frac{\eta_{R, D}}{\lambda_{R, D}}\right)
$$

where ${ }_{2}^{F} 1(a, b ; c ; z)$ is the Gaussian Hypergeometric function [36].

Proof:

The $\eta_{S, D}$ CDF is computed as [35]:

$$
F_{\eta_{S, D}}\left(\eta_{S, D}\right)=\int_{0}^{\eta_{S, D}} f_{\eta_{S, D}}(y) d y
$$

where $f_{\eta_{S, D}}(y)$ is given in (4). 
The CDF of $\eta_{R, D}$ is established by taking the integral of the PDF of $\eta_{R, D}$ given in (5) as the following:

$$
F_{\eta_{R, D}}\left(\eta_{R, D}\right)=\int_{0}^{\eta_{R, D}} f_{\eta_{R, D}}(y) d y
$$

Solving the integrals in (13) and (14) using [36, (3.194.1)], the tight formula of the $\eta_{S, D}$ 's CDF is going to be as (11) and $\eta_{R, D}$ 's CDF is going to be as (12).

\subsection{BER analysis}

The BER of the IR system is designated as:

$$
p_{e}=\operatorname{Pr}\left(\eta_{S, D} \leq \gamma_{o}\right) \times P_{\text {div }}(e)+\left(1-\operatorname{Pr}\left(\eta_{S, D} \leq \gamma_{o}\right)\right) \times P_{\text {direct }}(e)
$$

Depending on whether the relay is going to help or not, (15) encloses the two unconditional BER cases. Specifically, (15)'s second segment is used for the BER when the destination relies only on the DT. However, the first segment of (15) is for the BER of the path between the relay and the destination, where the destination would be using MRC to combine both signals.

$P_{\text {div }}(e)$ is the average MRC error probability in the combined diversity communication of the paths: source- and relay- destination. $P_{\text {direct }}(e)$ is the destination's error probability only in the source-destination path. $P_{\text {direct }}(e)$ can be expressed as:

$$
P_{\text {direct }}(e)=\int_{0}^{\infty} P_{\text {direct }}(e \mid \eta) f_{\eta_{S, D}}\left(\eta \mid \eta_{S, D}>\gamma_{o}\right) d \eta_{S, D}
$$

where $P_{\text {direct }}(e \mid \eta)$ is the conditional error probability which equals $\alpha Q\left(\sqrt{\beta \eta_{S, D}}\right)$ with the constellation parameters $\alpha$ and $\beta$. For instance, for Binary Phase Shift Keying (BPSK), $\alpha=1$ and $\beta=2$, for M-PSK, $\alpha=1$ and $\beta=2 \sin \left(\frac{\pi}{M}\right)^{2}$ and for M-QAM, $\alpha=4$ and $\beta=\frac{3}{(M-1)}$ and $Q(x)=\frac{1}{\sqrt{2 \pi}} \int_{x}^{\infty} e^{\left(-\frac{y^{2}}{2}\right)} d y$ is the Gausian Q-function. $f_{\eta_{S, D}}\left(\eta \mid \eta_{S, D}>\gamma_{o}\right)$ is the conditional PDF of $\eta_{S, D}$ given that $\eta_{S, D}$ is above the threshold $\gamma_{o}$ to indicate that the DT is successful which is given as:

$$
f_{\eta_{S, D}}\left(\eta \mid \eta_{S, D}>\gamma_{o}\right)=\left\{\begin{array}{cl}
0 & , \eta \leq \gamma_{o} \\
\rho \sum_{k=1}^{L} \pi_{k} \lambda_{S, D}\left[\frac{\eta_{S, D}^{m-1}}{\left(\eta_{S, D}+\lambda_{S, D}\right)^{m+1}}\right] & , \eta \geq \gamma_{o}
\end{array}\right.
$$

where

$$
\rho=\frac{(m) !}{\left[\Gamma(m)-(m-1) ! \sum_{k=1}^{L} \pi_{k}\left(\frac{\gamma_{o}}{\lambda_{S, D}}\right)^{m}{ }_{2}{ }_{2}\left(m+1, m ; m+1 ;-\frac{\gamma_{O}}{\lambda_{S, D}}\right)\right]}
$$

The approximated expression of $P_{\text {direct }}(e)$ can be found by placing (17) into (16) and using the Prony estimation of the Q-function with [36, (3.353.1)], and it is given as:

$$
\begin{aligned}
& P_{\text {direct }}(e)=\alpha \rho \sum_{i=1}^{2} A_{i} \sum_{k=1}^{L} \pi_{k} \lambda_{S, D} \sum_{n=0}^{\infty} \frac{\left(-a_{i} \beta\right)^{n}}{n !} \\
& \times\left[\frac{e^{-(n+m) \gamma_{o}}}{m !} \sum_{j=1}^{m} \frac{(j-1) !(-n-m)^{m-j}}{\left(\gamma_{o}+\lambda_{S, D}\right)^{j}}-\frac{(-n-m)^{m}}{m !} e^{(n+m) \lambda_{S, D}} E_{i}\left[-(n+m)\left(\gamma_{o}+\lambda_{S, D}\right)\right]\right.
\end{aligned}
$$

where $E_{i}[x]$ is the exponential integral function $[36,(8.211 .1)]$ and the terms $A_{i}$ and $a_{i}$ are the Prony estimation parameters. Full derivation of $P_{\text {direct }}(e)$ is provided in appendix A. The expression of the error probability $P_{\text {div }}(e)$, when the relay is helping and the destination is using MRC, is given by:

$$
P_{\text {div }}(e)=p_{S R}(e) p_{x}(e)+\left(1-p_{S R}(e)\right) p_{c o m}(e)
$$

where $p_{S R}(e)$ represents the relay's error probability in the source-relay path and $p_{x}(e)$ is the destination's error probability when the relay decodes the signal ineffectively and it is restricted to be below 0.5 as stated in [21]. $p_{c o m}(e)$ is the destination's error probability when the relay correctly decodes the signal. 
The expression $\left(1-p_{S R}(e)\right)$ indicates successful decoding at the relay, which achieves spatial diversity at the destination when it uses MRC to combine both received signals. $p_{S R}(e)$ is expressed as [1]:

$$
p_{S R}(e)=\left\{\begin{array}{rr}
\frac{1}{2}\left[1-\mu\left(\frac{\alpha^{2} \overline{\gamma_{S, R}}}{2 m}\right) \sum_{k=0}^{m-1}\left(\begin{array}{cc}
2 k \\
k
\end{array}\right)\left(\frac{1-\mu^{2}\left(\frac{\alpha^{2} \overline{\gamma_{S, R}}}{2 m}\right)}{4}\right)^{k}\right] & \text { m is integer } \\
\frac{1}{2 \sqrt{\pi}} \frac{\sqrt{\frac{\alpha^{2} \overline{\gamma_{S, R}}}{2 m}}}{\left(1+\frac{\alpha^{2} \overline{\gamma_{S, R}}}{2 m}\right)} \frac{\Gamma\left(m+\frac{1}{2}\right)}{m+(1 / 2)} \times{ }_{2} F_{1}\left(1, m+\frac{1}{2} ; m+1 ;\left(\frac{m}{m+\frac{\alpha^{2} \overline{\gamma_{S, R}}}{2}}\right)\right) & m \text { is noninteger }
\end{array}\right.
$$

where $\mu\left(\frac{\alpha^{2} \overline{\gamma_{S, R}}}{2 m}\right) \triangleq \sqrt{\frac{\alpha^{2} \overline{\gamma_{S, R}} / 2}{m+\alpha^{2} \overline{\gamma_{S, R}} / 2}},{ }_{2} 1(a, b ; c ; z)$ is the Gaussian Hypergeometric function [36]. and $\Gamma(x)$ is the Gamma function [36], $\overline{\gamma_{S, R}}$ is the relay's average SNR in the source-relay link. $p_{c o m}(e)$ is calculated by:

$$
p_{c o m}(e)=\alpha \int_{0}^{\infty} f_{x}\left(x \mid \eta_{S, D} \leq \gamma_{O}\right) Q(\sqrt{\beta x}) d x
$$

where $x=\eta_{S, D}+\eta_{R, D}$. Since $\eta_{S, D}$ is not comparable with $\eta_{R, D}$ at the MRC; thus, we will create a violation to work simply on $\eta_{R, D}$ and therefore, $p_{c o m}(e)$ will be given as:

$$
p_{\text {com }}(e) \approx \alpha \int_{0}^{\infty} f_{\eta_{R, D}}\left(\eta_{R, D} \mid \eta_{S, D} \leq \gamma_{O}\right) Q\left(\sqrt{\beta \eta_{R, D}}\right) d \eta_{R, D}
$$

where

$$
f_{\eta_{R, D}}\left(\eta_{R, D} \mid \eta_{S, D} \leq \gamma_{O}\right)=f_{\eta_{R, D}}\left(\eta_{R, D}\right)=\frac{(m) !}{\Gamma(m)} \sum_{k=1}^{L} \pi_{k}\left[\frac{\lambda_{R, D} \eta_{R, D}^{m-1}}{\left(\eta_{R, D}+\lambda_{R, D}\right)^{m+1}}\right]
$$

assuming that we have independent random variables [35].

The approximated expression of $p_{\text {com }}(e)$ is found by solving (22) utilizing the Prony estimation of the Q-function with [36, (3.353.3)], and it is given as:

$$
p_{c o m}(e) \approx \frac{\alpha m !}{\sqrt{2 \pi} \Gamma(1+m) \Gamma(m)}\left(\frac{2}{\beta}\right)^{m} \sum_{k=1}^{L} \pi_{k}\left(\lambda_{R, D}\right)^{-m} G_{3,2}^{1,3}\left(\frac{2}{\beta \lambda_{R, D}} \mid \begin{array}{c}
-m, 1-m, \frac{1}{2}-m \\
0,-m
\end{array}\right)
$$

where $G_{p, q}^{m, n}\left(\left.z\right|_{b} ^{a}\right)$ is the Meijer G function [37]. Full derivation of $p_{c o m}(e)$ is provided in appendix B. Equations (24), (20) and (18) can be substituted into (15) to get the expression of the BER.

\subsection{OP analysis}

Two events can properly describe the OP of the IR; when the DT is unsuccessful, a negative feedback will be sent to the relay in order to request the source's signal in the second time slot, then, the destination will use MRC to enhance the received signal. However, in the second time slot, there's still a chance for the received signal to be in outage even if the relay is helping, and that is when the two combined signals SNR are below the pre-defined threshold $\gamma_{o}$.

Both events of the IR's OP are represented by $p_{\text {out }}$, as the following:

$$
\begin{aligned}
p_{\text {out }} & =\operatorname{Pr}\left(\eta_{S, D} \leq \gamma_{o}\right) \times P_{r}\left[\min \left(\gamma_{S, R}, \eta_{S, D}+\eta_{R, D} \leq \gamma_{o} \mid \eta_{S, D} \leq \gamma_{o}\right)\right] \\
& =P_{r}\left(\eta_{S, D} \leq \gamma_{o}\right)\left[1-P_{r}\left(\eta_{S, D}+\eta_{R, D}>\gamma_{o} \mid \eta_{S, D} \leq \gamma_{o}\right)\right] \times P_{r}\left(\gamma_{S, R}>\gamma_{o} \mid \eta_{S, D} \leq \gamma_{o}\right) \\
& =P_{r}\left(\eta_{S, D} \leq \gamma_{o}\right) \times\left[1-\frac{P_{r}\left(\eta_{S, D} \leq \gamma_{o}\right)-P_{r}\left(\eta_{S, D}+\eta_{R, D} \leq \gamma_{o}\right)}{P_{r}\left(\eta_{S, D} \leq \gamma_{o}\right)} P_{r}\left(\gamma_{S, R}>\gamma_{o}\right)\right]
\end{aligned}
$$

It is worth to mentioning that the two OP events are contained in the operator $\min (\bullet \cdot \bullet)$, i.e., the first term is the relay's outage, however, the second term signifies the destination's outage. By simplifying the previous formula, we get:

$$
p_{\text {out }}=\operatorname{Pr}\left(\gamma_{S, R} \leq \gamma_{o}\right) \operatorname{Pr}\left(\eta_{S, D} \leq \gamma_{o}\right)+\operatorname{Pr}\left(\gamma_{S, R}>\gamma_{o}\right) \operatorname{Pr}\left(\eta_{S, D}+\eta_{R, D} \leq \gamma_{o}\right)
$$


When the relay is helping, the MRC is used at the destination. At that time, the value of $\eta_{S, D}$ is going to be much less than $\eta_{R, D}$; because of the diffeciencies of the DT. Intuitively, this leads to omitting $\eta_{S, D}$ from (26) which yields to the approximated OP expression, as the following:

$$
\begin{aligned}
p_{\text {out }} & \approx \frac{\gamma\left(m, m \frac{\gamma_{o}}{\gamma_{S, R}}\right)}{\Gamma(m)} \frac{(m-1) !}{\Gamma(m)} \sum_{k=1}^{L} \pi_{k}\left(\frac{\gamma_{o}}{\lambda_{S, D}}\right)^{m}{ }_{2} F_{1}\left(m+1, m ; m+1 ;-\frac{\gamma_{o}}{\lambda_{S, D}}\right) \\
& +\left[1-\frac{\gamma\left(m, m \frac{\gamma_{o}}{\bar{\gamma}_{S, R}}\right)}{\Gamma(m)}\right] \frac{(m-1) !}{\Gamma(m)} \sum_{k=1}^{L} \pi_{k}\left(\frac{\gamma_{o}}{\lambda_{R, D}}\right)^{m}{ }_{2} F_{1}\left(m+1, m ; m+1 ;-\frac{\gamma_{o}}{\lambda_{R, D}}\right)
\end{aligned}
$$

where $\mathrm{M}=\mathrm{L}$ is the number of interferers close to the destination, $\gamma(\mathrm{a}, \mathrm{b})$ is the Incomplete Gamma function [36].

\section{RESULTS AND DISCUSSION}

In this part of the paper, the performance of a DF-IR system in Nakagami-m fading environment with unique interferers near the destination is examined. Varying L along with $\gamma_{o}$ and interferers $\gamma$ 's would impact the system's performance; this is clarified by showing which parameter has affected the performance more severely. Figure 2 displays the influence of altering the threshold value on the BER for BPSK modulation with three interferers using $\mathrm{m}=3$ and $\gamma_{1}=7 \mathrm{~dB}, \gamma_{2}=10 \mathrm{~dB}$, and $\gamma_{3}=13 \mathrm{~dB}$. From this figure, if the threshold value decreases, then the system's BER will be enhanced.

Figure 3 shows the impact of altering $\gamma_{o}$ and the interferers $\gamma$ 's on the BER for BPSK modulation with three interferers $(\mathrm{L}=3)$ and fading severity parameter $m=3$. In this figure, if the threshold values increase, then the system's performance is going to be diminished. For instance, a system with $\left(\gamma_{o}=10 \mathrm{~dB}\right.$, $\gamma_{1}=7 \mathrm{~dB}, \gamma_{2}=10 \mathrm{~dB}$ and $\left.\gamma_{3}=13 \mathrm{~dB}\right)$ will act worse than a system with $\left(\gamma_{o}=10 \mathrm{~dB}, \gamma_{1}=2 \mathrm{~dB}, \gamma_{2}=5 \mathrm{~dB}\right.$ and $\left.\gamma_{3}=7 \mathrm{~dB}\right)$.

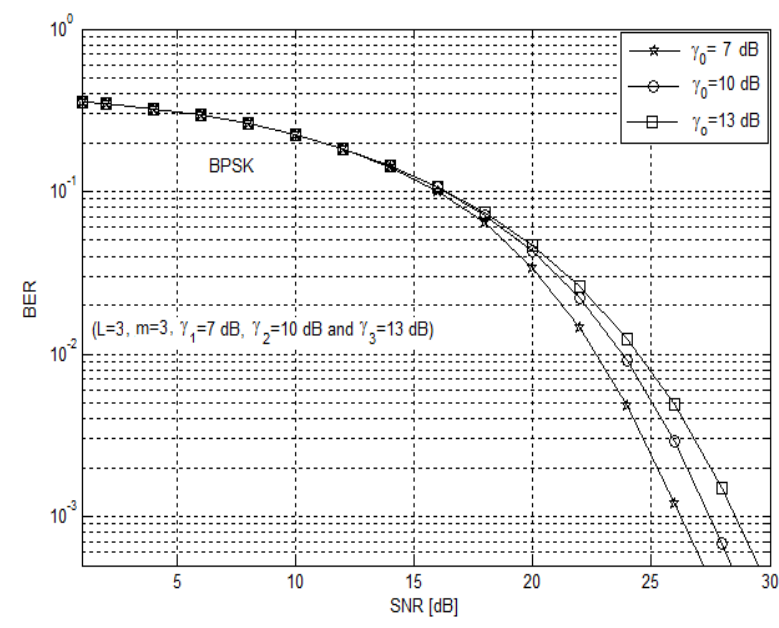

Figure 2. BER for BPSK modulation for various $\gamma_{0}$ 's and $\left(\mathrm{L}=3, \mathrm{~m}=3, \gamma_{1}=7 \mathrm{~dB}, \gamma_{2}=10 \mathrm{~dB}\right.$, and $\left.\gamma_{3}=13 \mathrm{~dB}\right)$

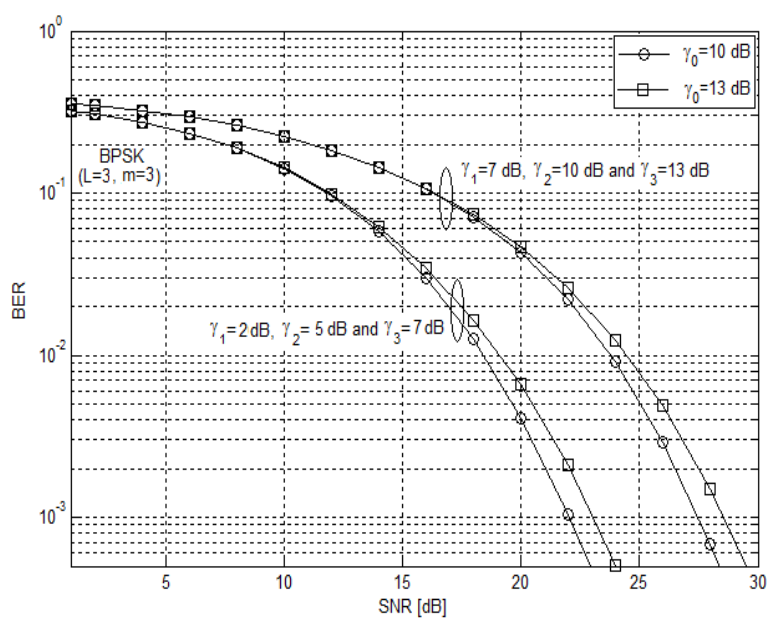

Figure 3. BER for BPSK modulation for various interferers $\gamma$ 's and, $(\mathrm{L}=\mathrm{m}=3)$

Figure 4 defines the effects of increasing $\mathrm{L}$ on the BER of the system for BPSK using $\mathrm{m}=3, \gamma_{o}=10$ $\mathrm{dB}, \gamma_{1}=7 \mathrm{~dB}, \gamma_{2}=10 \mathrm{~dB}$, and $\gamma_{3}=13 \mathrm{~dB}$. From this figure, as L increases, the system's BER is going to be severely degraded in reflection to the increase in co-channel interference. To demonstrate this, we say, for a $10^{-2}$ BER, a system with $(\mathrm{L}=1)$ is better than a system with $(\mathrm{L}=3)$ by $(17 \mathrm{~dB})$.

Figure 5 displays the BER against $\mathrm{SNR}$ of different $\mathrm{m}$ values with three destination-interferers and $\gamma_{o}=10 \mathrm{~dB}, \gamma_{1}=7 \mathrm{~dB}, \gamma_{2}=10 \mathrm{~dB}$, and $\gamma_{3}=13 \mathrm{~dB}$. In the figure, the system's BER improves, when the fading severity parameter $(\mathrm{m})$ increases. To show this, we say, for a $10^{-2}$ BER, a system with $(\mathrm{m}=4)$ transcends a system with $(\mathrm{m}=2)$ by $(2 \mathrm{~dB})$. 


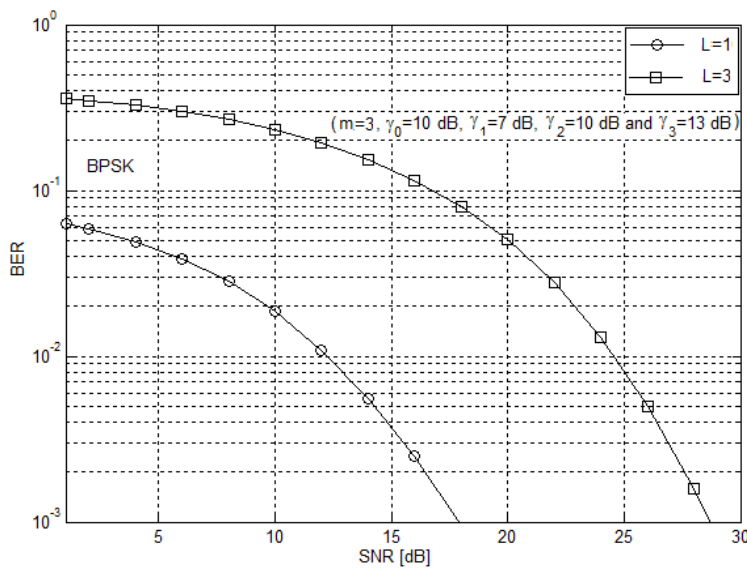

Figure 4. BER for BPSK modulation for various numbers of interferers $(\mathrm{L}=1,3), \mathrm{m}=3, \gamma_{o}=10 \mathrm{~dB}$, $\gamma_{1}=7 \mathrm{~dB}, \gamma_{2}=10 \mathrm{~dB}$, and $\gamma_{3}=13 \mathrm{~dB}$

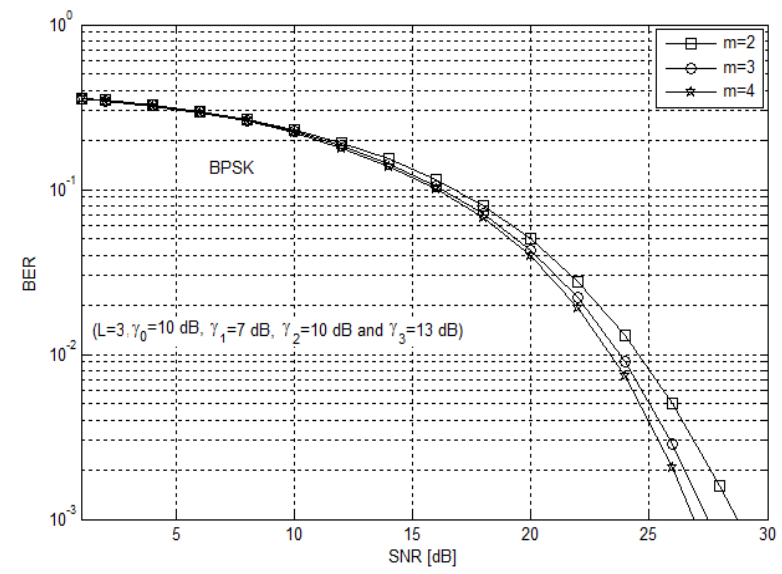

Figure 5. BER for various $m$ values and, $(\mathrm{L}=3$, $\gamma_{o}=10 \mathrm{~dB}, \gamma_{1}=7 \mathrm{~dB}, \gamma_{2}=10 \mathrm{~dB}$, and $\gamma_{3}=13 \mathrm{~dB}$ )

As Figure 6 illustrates, as $\gamma_{o}$ decreases, the OP will be upgraded. For example, given that the OP is 0.25 , a system with $\gamma_{o}=13 \mathrm{~dB}$ would need to increase about $6 \mathrm{~dB}$ to act like another one with $\gamma_{o}=7 \mathrm{~dB}$. This behavior happens for the following reason; as $\gamma_{o}$ decreases, the source-destination link is going to be less likely in outage and the relay-destination link is going to be used less frequently. Figure 7 shows the effect of the interferers $\gamma$ 's on the outage probability. From the figure, one can see that a system with $\left(\gamma_{o}=10 \mathrm{~dB}, \gamma_{1}=10 \mathrm{~dB}, \gamma_{2}=13 \mathrm{~dB}\right.$ and $\left.\gamma_{3}=15 \mathrm{~dB}\right)$ will act worse than a system with $\left(\gamma_{o}=10 \mathrm{~dB}, \gamma_{1}=2 \mathrm{~dB}, \gamma_{2}=5\right.$ $\mathrm{dB}$ and $\left.\gamma_{3}=7 \mathrm{~dB}\right)$.

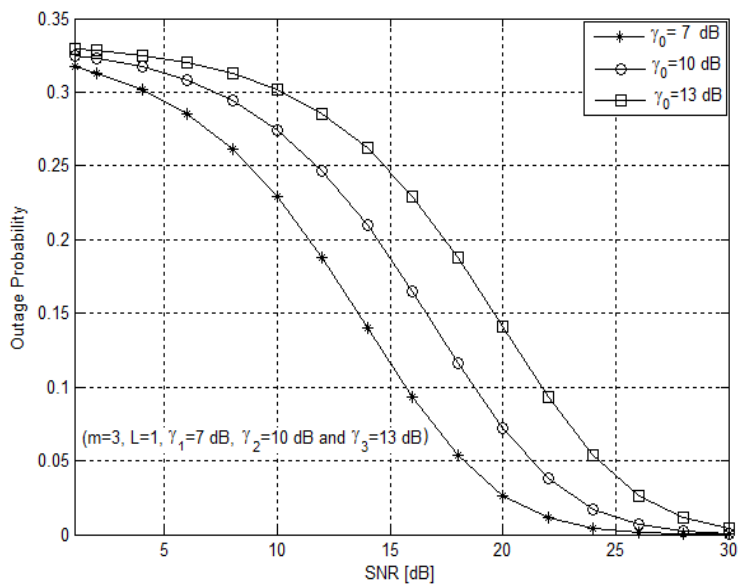

Figure 6. OP for various values of $\gamma_{o}$, and $(\mathrm{L}=1$, $\mathrm{m}=3, \gamma_{1}=7 \mathrm{~dB}, \gamma_{2}=10 \mathrm{~dB}$ and $\left.\gamma_{3}=13 \mathrm{~dB}\right)$

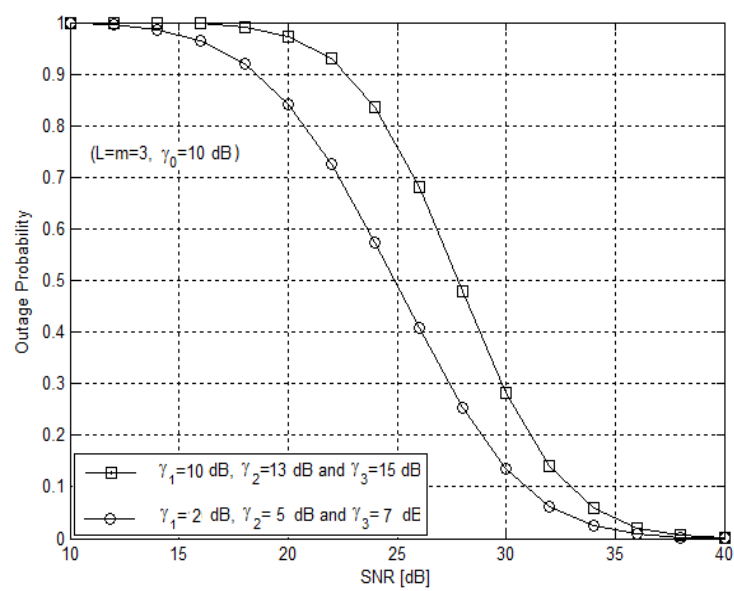

Figure 7. OP for various values of interferers $\gamma$ 's and $\left(\mathrm{L}=\mathrm{m}=3, \gamma_{o}=10 \mathrm{~dB}\right)$

Figure 8 shows the situation where we got several interferers close to the destination; clearly, the co-channel interference is going to increase the system's OP as L increases. For instance, we might compare two systems, one with $(\mathrm{L}=3)$ and the other with $(\mathrm{L}=1)$, and $\left(\gamma_{o}=10 \mathrm{~dB}, \gamma_{1}=7 \mathrm{~dB}, \gamma_{2}=10 \mathrm{~dB}\right.$ and $\gamma_{3}=13 \mathrm{~dB}$ ) for the OP of 0.3 , there's about $20 \mathrm{~dB}$ difference in the SNR which confirms that the co-channel interference is going to impair the system's performance.

Figure 9 illustrates the OP versus SNR for various values of $\mathrm{m}$ with one interferer and $\gamma_{o}=10 \mathrm{~dB}$, $\gamma_{1}=7 \mathrm{~dB}, \gamma_{2}=10 \mathrm{~dB}$, and $\gamma_{3}=13 \mathrm{~dB}$. As realized in this figure, the increment of the fading severity parameter $(\mathrm{m})$ is going to enhance the system's performance by means of OP. For instance, for OP of 0.1, a system with $(\mathrm{m}=4)$ surpasses a different one with $(\mathrm{m}=1)$ by $(3 \mathrm{~dB})$. 


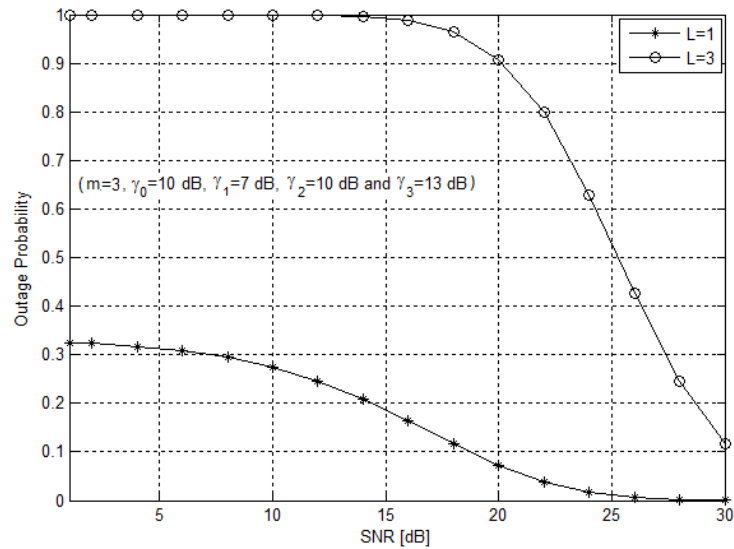

Figure 8. OP for various values of $\mathrm{L}$ and $(\mathrm{m}=3$, $\gamma_{o}=10 \mathrm{~dB}, \gamma_{1}=7 \mathrm{~dB}, \gamma_{2}=10 \mathrm{~dB}$ and $\left.\gamma_{3}=13 \mathrm{~dB}\right)$

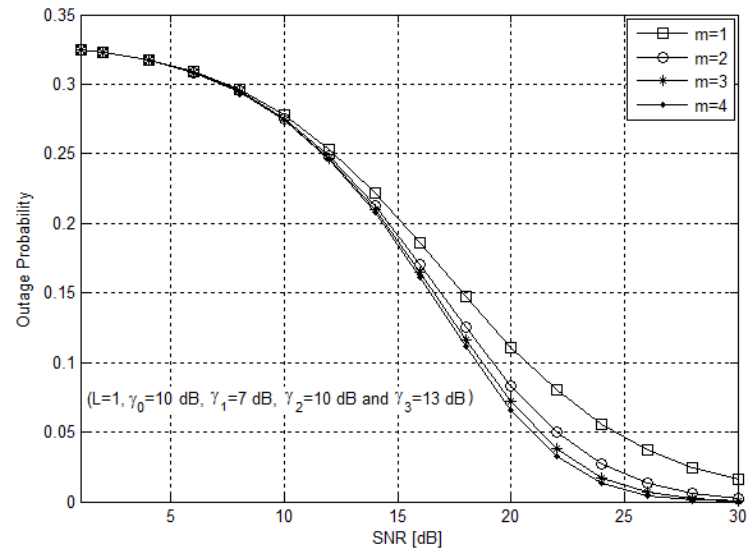

Figure 9. OP for various values of $\mathrm{m}$ and, $(\mathrm{L}=1$, $\gamma_{o}=10 \mathrm{~dB}, \gamma_{1}=7 \mathrm{~dB}, \gamma_{2}=10 \mathrm{~dB}$, and $\left.\gamma_{3}=13 \mathrm{~dB}\right)$

\section{CONCLUSION}

The performance of a DF-IR system throughout a Nakagami-m fading channel with unique interferers near the destination was evaluated. The evaluation clarified the impact of the change in the threshold $\gamma_{o}$ and in the co-channel interference on the system's BER and OP. Efficient use of the channel's bandwidth is guaranteed by achieving spatial diversity in IR systems. A derivation of the BER and OP formulas was proposed in this study. Outcomes have shown that if the order of the Nakagami-m fading channel decreases and the number of interferes increases, the system's BER and OP will get weakened. Moreover, there is a huge effect of $\gamma_{o}$ and the interferers $\gamma$ 's on the performance of the analyzed system.

\section{APPENDIX}

\section{A. Derivation of $\boldsymbol{P}_{\text {direct }}(\boldsymbol{e})$}

The expression of $P_{\text {direct }}(e)$ is denoted as:

$$
P_{\text {direct }}(e)=\int_{0}^{\infty} P_{\text {direct }}(e \mid \eta) f_{\eta_{S, D}}\left(\eta \mid \eta_{S, D}>\gamma_{o}\right) d \eta
$$

The conditional PDF $f_{\eta_{S, D}}\left(\eta \mid \eta_{S, D}>\gamma_{o}\right)$ is given as:

$$
f_{\eta_{S, D}}\left(\eta \mid \eta_{S, D}>\gamma_{o}\right)=\frac{f_{\eta_{S, D}}\left(\eta_{S, D}\right)}{1-F_{\eta_{S, D}}\left(\gamma_{o}\right)}=\left\{\begin{array}{cc}
0 & , \eta \leq \gamma_{o} \\
\rho \sum_{k=1}^{L} \pi_{k} \lambda_{S, D}\left[\frac{\eta_{S, D}^{m-1}}{\left(\eta_{S, D}+\lambda_{S, D}\right)^{m+1}}\right] & , \eta \geq \gamma_{o}
\end{array}\right.
$$

where

$\rho=\frac{(m) !}{\left[\Gamma(m)-(m-1) ! \sum_{k=1}^{L} \pi_{k}\left(\frac{\gamma_{o}}{\lambda_{S, D}}\right)^{m}{ }_{2}{ }_{2}\left(m+1, m ; m+1 ;-\frac{\gamma_{o}}{\lambda_{S, D}}\right)\right]}$

Substitution (A.2) into (A.1) to get

$$
\begin{aligned}
P_{\text {direct }}(e) & =\int_{\gamma_{o}}^{\infty} \alpha Q\left(\sqrt{\beta \eta_{S, D}}\right) \rho \sum_{k=1}^{L} \pi_{k} \lambda_{S, D}\left[\frac{\eta_{S, D}^{m-1}}{\left(\eta_{S, D}+\lambda_{S, D}\right)^{m+1}}\right] d \eta_{S, D} \\
& =\alpha \rho \sum_{k=1}^{L} \pi_{k} \lambda_{S, D} \int_{\gamma_{o}}^{\infty} Q\left(\sqrt{\beta \eta_{S, D}}\right)\left[\frac{\eta_{S, D}^{m-1}}{\left(\eta_{S, D}+\lambda_{S, D}\right)^{m+1}}\right] d \eta_{S, D}
\end{aligned}
$$

Using the Prony's approximation of the Q-function 


$$
Q(x) \approx \sum_{i=1}^{p} A_{i} e^{-a_{i} x^{q}}
$$

where $x=\sqrt{\beta \eta_{S, D}}, p=q=2, A_{1}=0.208, A_{2}=0.147, a_{1}=0.971, a_{2}=0.525$.

$P_{\text {direct }}(e)$ becomes:

$$
\begin{aligned}
P_{\text {direct }}(e) & =\alpha \rho \int_{\gamma_{o}}^{\infty} \sum_{i=1}^{p} A_{i} e^{-a_{i} \beta \eta_{S, D}} \sum_{k=1}^{L} \pi_{k} \lambda_{S, D}\left[\frac{\eta_{S, D}^{m-1}}{\left(\eta_{S, D}+\lambda_{S, D}\right)^{m+1}}\right] d \eta_{S, D} \\
& =\alpha \rho \sum_{i=1}^{2} A_{i} \sum_{k=1}^{L} \pi_{k} \lambda_{S, D} \int_{\gamma_{o}}^{\infty}\left[\frac{\eta_{S, D}^{m-1} e^{-a_{i} \beta \eta_{S, D}}}{\left(\eta_{S, D}+\lambda_{S, D}\right)^{m+1}}\right] d \eta_{S, D}
\end{aligned}
$$

Define:

$$
I=\int_{\gamma_{o}}^{\infty}\left[\frac{\eta_{S, D}^{m-1} e^{-a_{i} \beta \eta_{S, D}}}{\left(\eta_{S, D}+\lambda_{S, D}\right)^{m+1}}\right] d \eta_{S, D}
$$

Using [36, (3.353.1)], I will be as:

$$
I=\sum_{n=0}^{\infty} \frac{\left(-a_{i} \beta\right)^{n}}{n !}\left[\frac{e^{-(n+m) \gamma_{o}}}{m !} \sum_{j=1}^{m} \frac{(j-1) !(-n-m)^{m-j}}{\left(\gamma_{o}+\lambda_{S, D}\right)^{j}}-\frac{(-n-m)^{m}}{m !} e^{(n+m) \lambda_{S, D}} E_{i}\left[-(n+m)\left(\gamma_{o}+\lambda_{S, D}\right)\right]\right] \text { (A.8) }
$$

Substituting (A.8) into (A.6), the approximated expression of $P_{\text {direct }}(e)$ is given as:

$$
\begin{aligned}
& P_{\text {direct }}(e)=\alpha \rho \sum_{i=1}^{2} A_{i} \sum_{k=1}^{L} \pi_{k} \lambda_{S, D} \sum_{n=0}^{\infty} \frac{\left(-a_{i} \beta\right)^{n}}{n !} \times\left[\frac{e^{-(n+m) \gamma_{o}}}{m !} \sum_{j=1}^{m} \frac{(j-1) !(-n-m)^{m-j}}{\left(\gamma_{o}+\lambda_{S, D}\right)^{j}}-\right. \\
& \left.\frac{(-n-m)^{m}}{m !} e^{(n+m) \lambda_{S, D}} E_{i}\left[-(n+m)\left(\gamma_{o}+\lambda_{S, D}\right)\right]\right]
\end{aligned}
$$

where $E_{i}[x]$ is the exponential integral function [36, (8.211.1)] and the terms $A_{i}$ and $a_{i}$ are the Prony approximation parameters.

\section{B. Derivation of $p_{\text {com }}(e)$}

The expression of $p_{c o m}(e)$ is denoted as:

$$
\begin{aligned}
& p_{c o m}(e)=\alpha \int_{0}^{\infty} f_{x}\left(x \mid \eta_{S, D} \leq \gamma_{O}\right) Q(\sqrt{\beta x}) d x \\
& x=\eta_{S, D}+\eta_{R, D}
\end{aligned}
$$

A transgression will be made on the random variable $x$ to work merely on $\eta_{R, D}$. Since $\eta_{R, D}$ will be much greater than $\eta_{S, D}$ to get the approximated expression of $p_{c o m}(e)$ as follows:

$$
p_{\text {com }}(e) \approx \alpha \int_{0}^{\infty} f_{\eta_{R, D}}\left(\eta_{R, D} \mid \eta_{S, D} \leq \gamma_{O}\right) Q(\sqrt{\beta \eta}) d \eta_{R, D}
$$

The conditional PDF $f_{\eta_{R, D}}\left(\eta_{R, D} \mid \eta_{S, D} \leq \gamma_{O}\right)$ is given as:

$$
f_{\eta_{R, D}}\left(\eta_{R, D} \mid \eta_{S, D} \leq \gamma_{O}\right)=f_{\eta_{R, D}}\left(\eta_{R, D}\right)=\frac{(m) !}{\Gamma(m)} \sum_{k=1}^{L} \pi_{k}\left[\frac{\lambda_{R, D} \eta_{R, D}^{m-1}}{\left(\eta_{R, D}+\lambda_{R, D}\right)^{m+1}}\right]
$$

Substituting (B.3) into (B.2):

$$
\begin{aligned}
p_{c o m}(e) & \approx \alpha \int_{0}^{\infty} Q\left(\sqrt{\beta \eta_{R, D}}\right) \frac{(m) !}{\Gamma(m)} \sum_{k=1}^{L} \pi_{k} \lambda_{R, D}\left[\frac{\eta_{R, D}^{m-1}}{\left(\eta_{R, D}+\lambda_{R, D}\right)^{m+1}}\right] d \eta_{R, D} \\
& \approx \alpha \frac{(m) !}{\Gamma(m)} \sum_{k=1}^{L} \pi_{k} \lambda_{R, D} \int_{0}^{\infty} Q\left(\sqrt{\beta \eta_{R, D}}\right)\left[\frac{\eta_{R, D}^{m-1}}{\left(\eta_{R, D}+\lambda_{R, D}\right)^{m+1}}\right] d \eta_{R, D}
\end{aligned}
$$

Define: 
$I=\int_{0}^{\infty} Q\left(\sqrt{\beta \eta_{R, D}}\right)\left[\frac{\eta_{R, D}^{m-1}}{\left(\eta_{R, D}+\lambda_{R, D}\right)^{m+1}}\right] d \eta_{R, D}$

Using

$$
\begin{aligned}
& Q(\sqrt{\alpha \gamma})=\frac{1}{\sqrt{2 \pi}} G_{1,2}^{2,0}\left(\frac{\alpha \gamma}{2} \mid \begin{array}{c}
1 \\
0, \frac{1}{2}
\end{array}\right) \\
& \frac{1}{\left(1+\frac{\gamma}{\alpha}\right)^{M}}=\frac{1}{\Gamma(M)} G_{1,1}^{1,1}\left(\begin{array}{c|c}
\frac{\gamma}{\alpha} & 1-M \\
0
\end{array}\right)
\end{aligned}
$$

Based on (B.6) and (B.7),

$$
\begin{aligned}
& Q\left(\sqrt{\beta \eta_{R, D}}\right)=\frac{1}{\sqrt{2 \pi}} G_{1,2}^{2,0}\left(\frac{\beta \eta_{R, D}}{2} \mid \begin{array}{c}
1 \\
0, \frac{1}{2}
\end{array}\right) \\
& \frac{1}{\left(\eta_{R, D}+\lambda_{R, D}\right)^{m+1}}=\frac{1}{\left(\lambda_{R, D}\right)^{m+1}} \frac{1}{\left(1+\frac{\eta_{R, D}}{\lambda_{R, D}}\right)^{m+1}}=\frac{1}{\left(\lambda_{R, D}\right)^{m+1}} \frac{1}{\Gamma(m+1)} G_{1,1}^{1,1}\left(\frac{\eta_{R, D}}{\lambda_{R, D}} \mid \begin{array}{c}
-m \\
0
\end{array}\right)
\end{aligned}
$$

Substituting (B.5), (B.8), and (B.9) into (B.4), we get:

$$
\begin{aligned}
p_{\text {com }}(e) & \approx \alpha \frac{(m) !}{\Gamma(m)} \frac{1}{\sqrt{2 \pi}} \frac{1}{\Gamma(m+1)} \sum_{k=1}^{L} \pi_{k} \lambda_{R, D} \frac{1}{\left(\lambda_{R, D}\right)^{m+1}} \int_{0}^{\infty} \eta_{R, D}^{m-1} G_{1,2}^{2,0}\left(\frac{\beta \eta_{R, D}}{2} \mid \begin{array}{c}
1 \\
0, \frac{1}{2}
\end{array}\right) G_{1,1}^{1,1}\left(\frac{\eta_{R, D}}{\lambda_{R, D}} \mid \begin{array}{c}
-m \\
0
\end{array}\right) d \eta_{R, D} \\
& \approx \frac{\alpha(m) !}{\sqrt{2 \pi} \Gamma(m) \Gamma(m+1)} \sum_{k=1}^{L} \pi_{k} \lambda_{R, D}^{-m} \int_{0}^{\infty} \eta_{R, D}^{m-1} G_{1,2}^{2,0}\left(\frac{\beta \eta_{R, D}}{2} \mid \begin{array}{c}
1 \\
0, \frac{1}{2}
\end{array}\right) G_{1,1}^{1,1}\left(\frac{\eta_{R, D}}{\lambda_{R, D}} \mid \begin{array}{c}
-m \\
0
\end{array}\right) d \eta_{R, D}
\end{aligned}
$$

Using [37, $(07.34 .21 .0011 .01)]$, the approximated expression of $p_{c o m}(e)$ is given as:

$$
p_{\text {com }}(e) \approx \frac{\alpha(m) !}{\sqrt{2 \pi} \Gamma(m) \Gamma(m+1)}\left(\frac{2}{\beta}\right)^{m} \sum_{k=1}^{L} \pi_{k} \lambda_{R, D}^{-m} G_{3,2}^{1,3}\left(\frac{2}{\beta \lambda_{R, D}} \mid \begin{array}{c}
-m, 1-m, \frac{1}{2}-m \\
0,-m
\end{array}\right)
$$

\section{REFERENCES}

[1] M. O. Hasna and M. S. Alouini, "End-to-end performance of transmission systems with relays over Rayleighfading channels," IEEE Transactions on Wireless Communications, vol. 2, no. 6, pp. 1126-1131, 2003.

[2] K. A. Darabkh, et al., "BPA-CRP: A Balanced Power-Aware Clustering and Routing Protocol for Wireless Sensor Networks," Ad Hoc Networks, vol. 82, pp. 155-171, Jan. 2019.

[3] K. A. Darabkh, et al., "A generic buffer occupancy expression for stop-and-wait hybrid automatic repeat request protocol over unstable channels," Telecommunication Systems, vol. 63, no. 2, pp. 205-221, 2016.

[4] K. A. Darabkh, "Fast and Upper Bounded Fano Decoding Algorithm: Queuing Analysis," Transactions on Emerging Telecommunications Technologies, vol. 28, no. 1, pp. 1-12, 2017.

[5] K. A. Darabkh, "Evaluation of Channel Adaptive Access Point System with Fano Decoding," International Journal of Computer Mathematics, vol. 88, no. 5, pp. 916-937, 2011.

[6] K. A. Darabkh, et al., "New Arriving Process for Convolutional Codes with Adaptive Behavior," International Multi-conference on Systems, Signals, and Devices, Chemnitz, Germany, pp. 1-6, 2012.

[7] K. A. Darabkh and B. N. Abu-Jaradeh, "Buffering Study over Intermediate Hops including Packet Retransmission," 2010 International Conference on Multimedia Computing and Information Technology (MCIT2010), Sharjah, U.A.E, pp. 45-48, 2010.

[8] K. A. Darabkh and B. N. Abu-Jaradeh, "Bounded Fano Decoders over Intermediate Hops Excluding Packet Retransmission," 2010 24th International Conference on Advanced Information Networking and Applications (AINA 2010), Perth, Australia, pp. 299-303, 2010.

[9] K. A. Darabkh and W. D. Pan, "Queueing Simulation for Fano Decoders with Finite Buffer Capacity," Proceedings of the 9th Communications and Networking Simulation Symposium Conference (CNSS'06), Huntsville, Alabama, Apr. 2006.

[10] R. T. Al-zubi, et al., "Outage Performance of Cognitive Two-Way Amplify-and-Forward Relay Network under Different Transmission Schemes," Transactions on Emerging Telecommunications Technologies, 2020.

[11] R. Saifan, et al., "Probabilistic and Deterministic Path Selection in Cognitive Radio Network," IET Communications, vol. 13, no. 17, p. 2767-2777, Oct. 2019.

[12] K. A. Darabkh, et al., "A-Z Overview of the In-Band Full-Duplex Cognitive Radio Networks," Computer Communications, vol. 145, pp. 66-95, Sep. 2019. 
[13] F. H. P. Fitzek and M. D. Katz, "Cooperation in wireless networks: principles and applications," Springer, 2006.

[14] M. K. Simon and M. S. Alouini, "Digital Communication over Fading Channels," John Wiley and Sons, New York, NY, USA, 2000.

[15] J. N. Laneman, et al., "Cooperative diversity in wireless networks: efficient protocols and outage behavior," IEEE Transactions on Information Theory, vol. 50, no. 12, pp. 3062-3080, Dec. 2004.

[16] Z. Yi and I. M. Kim, "Diversity order analysis of the decode-and-forward cooperative networks with relay selection," IEEE Transactions on Wireless Communications, vol. 7, no. 5, pp. 1792-1799, 2008.

[17] X. Zhang, et al., "Performance analysis of relay assignment schemes for cooperative networks with multiple source-destination pairs," IEEE Transactions on Wireless Communications, vol. 11, no. 1, pp. 166-177, 2012.

[18] P. A. Anghel and M. Kaveh, "Exact symbol error probability of a cooperative network in a Rayleigh-fading environment," IEEE Transactions on Wireless Communications, vol. 3, no. 5, pp. 1416-1421, Sep. 2004.

[19] M. F. Al-Mistarihi, et al., "Performance evaluation of multiuser diversity in multiuser two-hop cooperative multirelay wireless networks using maximal ratio combining over Rayleigh fading channels," International Journal of Communication Systems, vol. 28, no. 1, pp. 71-90, 2015.

[20] M. M. Harb and M. F. Al-Mistarihi, "Dual Hop Differential Amplify-and-Forward Relaying with Selection Combining Cooperative Diversity over Nakagami-m Fading Channels," 2016 8th IEEE International Conference on Communication Software and Networks (ICCSN), Beijing, China, pp. 225-228, 2016.

[21] S. Al-Zoubi, et al., "On the Outage Probability of DF Relay Selection Cooperative Wireless Networks over Nakagami-m Fading Channels," 2016 7th International Conference on Information and Communication Systems (ICICS), Irbid, Jordan, 2016.

[22] M. F. Al-Mistarihi, et al., "BER Analysis in Dual Hop Differential Amplify-and-Forward Relaying Systems with Selection Combining Using M-ary Phase-Shift Keying over Nakagami-m Fading Channels," in Galinina O., et al. (eds), "Internet of Things, Smart Spaces, and Next Generation Networks and Systems," NEW2AN/ruSMART 2019, Lecture Notes in Computer Science, vol. 11660, 2019.

[23] M. F. Al-Mistarihi, et al., "Performance of Relay-Based Decode and Forward Cooperative Diversity Systems over Rayleigh Fading Channels with Non-Identical Interferers," IET Communications, vol. 13, no. 19, pp. 3135-3144, Nov. 2019.

[24] M. Hlayel, et al., "Closed-form Expression of Bit Error Rate in Dual-Hop Dual-Branch Mixed Relaying Cooperative Networks with Best-Path Selection over Rayleigh Fading Channels," 2014 IEEE 11th International Multi-Conference on Systems, Signals \& Devices (SSD 2014), Castelldefels-Barcelona, Spain, 2014.

[25] M. F. Al-Mistarihi, et al., "BER Analysis in Relay-Based DF Cooperative Diversity Systems over Rayleigh Fading Channels with Non-Identical Interferers near the Destination," 2019 International Conference on Advanced Communication Technologies and Networking (CommNet), Rabat, Morocco, pp. 1-5, 2019.

[26] S. S. Ikki and M. H. Ahmed, "Performance analysis of cooperative diversity wireless networks over Nakagami-m fading channel," IEEE Communications Letters, vol. 11, no. 4, pp. 334-336, Apr. 2007.

[27] R. Mohaisen and M. F. Al-Mistarihi, "Performance Evaluation of Bit Error Rate in Relay-Based Cooperative Diversity Systems over Rayleigh Fading Channels with Interference," 11th International Conference on Wired/Wireless Internet Communications (WWIC 2013), Saint-Petersburg, Russia, pp. 241-250, 2013.

[28] R. Mohaisen and M. F. Al-Mistarihi, "Closed-form Expression for Outage Probability in Relay-Based Cooperative Diversity Systems over Rayleigh Fading Channels with Interference," 2013 3rd International Conference on Communications and Information Technology (ICCIT 2013), Beirut, Lebanon, pp. 300-303, 2013.

[29] S. S. Ikki and M. H. Ahmed, "Performance of multiple-relay cooperative diversity systems with best relay selection over Rayleigh fading channels," EURASIP Journal on Advances in Signal Processing, 2008.

[30] S. S. Ikki and M. H. Ahmad, "Performance Analysis of Decode-and-Forward Incremental Relaying CooperativeDiversity Networks over Rayleigh Fading Channels," IEEE 69th Vehicular Technology Conference (VTC-Spring'09), Barcelona, Spain, pp. 1-6, 2009.

[31] A. M. Hayajneh, et al., "Incremental-Relaying Cooperative-Networks using Dual Transmit Diversity and Decode and Forward Relaying Scheme with Best Relay Selection," 2014 37th Jubilee International Convention on Information and Communication Technology, Electronics and Microelectronics (MIPRO), Opatija, Croatia, 2014.

[32] M. F. Al-Mistarihi, et al., "Closed-form Expression for Outage Probability in Relay-Based Cooperative Diversity Systems over Multipath Fading Channels with Interference," 2014 IEEE 11th International Multi-Conference on Systems, Signals \& Devices (SSD 2014), Castelldefels-Barcelona, Spain, pp. 1-5, 2014.

[33] A. Magableh, et al., "Closed-form Expression for Bit Error Rate in Relay-Based Cooperative Diversity Systems over Multipath Fading Channels with Interference," 2013 9th International Wireless Communications and Mobile Computing (IWCMC 2013), Sardinia, Italy, Jul. 2013.

[34] M. F. Al-Mistarihi, et al., "Closed-form Expression for BER in Relay-Based DF Cooperative Diversity Systems over Nakagami-m Fading Channels with Non-Identical Interferers," in Internet of Things, Smart Spaces, and Next Generation Networks and Systems, pp. 700-709, 2019.

[35] A. Papoulis and S. U. Pillai, "Probability, Random Variables, and Stochastic Processes," 4th edition, New York, McGraw-Hill, 2002.

[36] I. S. Gradshteyn and I. M. Ryzhik, "Table of Integrals, Series, and Products," 7th edition, Academic Press, 2007.

[37] Miejer"s G-Function, "The wolfram functions site," 2001. [Online]. Available: http://functions.wolfram.com/07.34.21.0011.01. 


\section{BIOGRAPHIES OF AUTHORS}
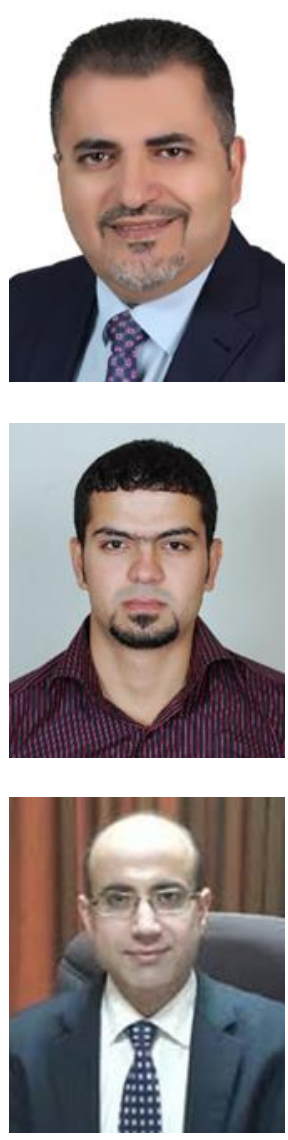

Mamoun F. Al-Mistarihi received the B.Sc. and M.Sc. degrees in Electrical Engineering from Jordan University of Science and Technology, Irbid, Jordan, M.E.E. and Ph.D. degrees in Electrical Engineering from University of Minnesota, Minneapolis, MN, USA, in 1992, 1996, 2005, and 2005, respectively. From 1994 to 2000, he was with the Royal Scientific Society, Amman, Jordan. Presently he is an Associate Professor with the Electrical Engineering Department, Jordan University of Science and Technology, Irbid, Jordan. His research interests include digital signal processing, image processing, digital signal processing for communications, wireless communications and mobile networks, performance evaluation of wireless communication systems over fading channels, security of wireless systems, WiMAX, wireless Ad Hoc networks, and wireless sensor networks.

Rami Mohaisen received the B.Sc. and M.Sc. degrees in Electrical Engineering from Jordan University of Science and Technology, Irbid, Jordan in 2010, and 2012, respectively. His research interests include wireless communications, and communication networks.

Khalid A. Darabkh received the PhD degree in Computer Engineering from the University of Alabama in Huntsville, USA, in 2007 with honors. He has joined the Computer Engineering Department at the University of Jordan as an Assistant Professor since 2007 and has been a Tenured Full Professor since 2016. He is engaged in research mainly on wireless sensor networks, mobile-ad hoc networks, vehicular networks, flying ad hoc networks, queuing systems and networks, multimedia transmission, Internet of things, cognitive radio networks, channel coding, steganography and watermarking, as well as innovative and interactive learning environments. He authored and co-authored of at least a hundred forty research articles and served as a reviewer in many scientific journals and international conferences. Prof. Darabkh is the recipient of 2016 Ali Mango Distinguished Researcher Reward for Scientific Colleges and Research Centers in Jordan. He is further the recipient of the University of Jordan's appreciation certificate as of being the researcher who publish the highest number of quality manuscripts in Scopus during 2018. As far as software certifications tracks are concerned, he is Sun Certified Programmer for Java Platform (SCJP), Oracle Database Administrator Certified Associate (OCA), as well as Oracle Java Developer Certified Trainer (OJDC-T). In fact, he serves on the Editorial Board of Telecommunication Systems, published by Springer, Computer Applications in Engineering Education, published by John Wiley \& Sons, and Journal of High Speed Networks, published by IOS Press. Additionally, he serves as a TPC member of highly reputable IEEE conferences such as GLOBECOM, LCN, VTC-Fall, PIMRC, ISWCS, ATC, ICT, and IAEAC. Moreover, he is a member of many professional and honorary societies, including Eta Kappa Nu, Tau Beta Pi, Phi Kappa Phi, and Sigma XI. He was selected for inclusion in the Who's Who Among Students in American Universities and Colleges and Marquis Who's Who in the World. As administrative experience at the University of Jordan, he served as Assistant Dean for Computer Affairs in the College of Engineering from Sept 2008 to Sept 2010. Additionally, he served as Acting Head of the Computer Engineering Department from June 2010 to Sept 2012. 